\title{
Efficient Multiway Relaying for Data Sharing in Energy Harvesting Sensor Networks
}

\author{
Moslem Noori and Masoud Ardakani \\ Department of Electrical and Computer Engineering, University of Alberta, 9107116 Street, Edmonton, AB, Canada T6G 2V4 \\ Correspondence should be addressed to Masoud Ardakani; ardakani@ualberta.ca
}

Received 4 November 2014; Revised 18 March 2015; Accepted 21 March 2015

Academic Editor: Mana Sriyudthsak

Copyright ( 2015 M. Noori and M. Ardakani. This is an open access article distributed under the Creative Commons Attribution License, which permits unrestricted use, distribution, and reproduction in any medium, provided the original work is properly cited.

In a wireless sensor network (WSN), sensors often need to share their measurements for applications like distributed estimation and detection or data aggregation. Here, we suggest using multiway relaying (MWR) for data sharing between energy harvesting sensors that cannot directly communicate with each other. We first start by studying the achievable data rate of amplify-and-forward (AF) MWR for energy harvesting sensors. Then, we show that, by backing off the transmit power at the sensors, not only better energy efficiency and longer lifetime are achieved, but also the data sharing rate increases. Based on this result, we further improve the performance of AF MWR in the assumed WSN by smartly adjusting the transmit power at the sensors. Our power allocation is devised in a way to improve the energy efficiency of MWR and increase the sum rate of data sharing between the sensors over the network lifetime. Simulation results are presented to verify the enhancement achieved by using our proposed power allocation technique.

\section{Introduction}

Sensors often disseminate and share data with each other to accomplish a collaborative task in a wireless sensor network (WSN) [1]. Such collaboration could lead to achieving a common goal in the network or improving its general performance. For instance, by sharing their measurement data, sensors are able to track moving objects in their vicinity or improve their estimation from a parameter to make further decisions $[2,3]$. Also, neighboring sensors may share their measurements to form an aggregated version of the data to be sent to the data sink.

Sharing data between the sensors can take place in different forms. Flooding [1] is one of the data sharing/dissemination approaches where a sensor broadcasts its own message or a message received from another sensor. This continues until the data is shared with all intended sensors. Flooding, however, could be inefficient due to its high energy consumption, coming from repetitive packet transmission by the sensors and possible collision between the flooded packets [4]. Another strategy is to take a cooperative approach $[5,6]$ where a central (relay) node is used to coordinate data sharing between the sensors [7]. In this approach, the relay node asks the sensors to transmit their data to it and then it distributes the data between the sensors. This approach is more efficient in terms of energy consumption at the cost of extra hardware and more complex implementation. In addition, several other studies have considered developing new techniques or variations of the flooding and cooperative schemes with better energy efficiency, for example, [8-10].

Multiway relaying (MWR) [11] is a new efficient data sharing paradigm for wireless networks. This approach is cooperative and a relay node is used to assist the users (sensors) to share their data. MWR can achieve better bandwidth/energy efficiency compared to conventional cooperative schemes. This is achieved through employing physical layer network coding [12] that smartly leverages interference between different users' transmitted signals. Despite many prior studies on the advantage of MWR including its achievable data rates and energy efficiency $[11,13-16]$, its application for WSNs has not been investigated.

In this paper, we study the application of amplify-andforward (AF) MWR for WSNs. More specifically, we consider a group of energy harvesting sensors that want to share their 
data with each other. The sensors do not have direct communication links, for example, due to surrounding obstacles; hence, employing a cooperative communication approach is essential. To this end, a relay node is used to assist the sensors with their data communication. For such a network, we first find the achievable rate of data sharing between sensors for a given time span using MWR. Then, we show that unlike conventional cooperative schemes for data sharing, by backing off the transmit power at the sensors, we can further improve the energy efficiency as well as the data rate of AF MWR.

Considering the above, an important question is how to assign the transmit power at the sensors to get the best performance out of the network. To address this, we formulate an optimization problem to maximize the data sharing sum rate in the network while achieving a target lifetime is guaranteed. For this optimization problem, we show that the number of constraints grows exponentially with the number of sensors. As a result, solving it becomes drastically complex as the number of sensors increases. That said, we identify the conditions in the problem that are more likely to be active and formulate a relaxed version of the problem based on them. While the number of constraints in the relaxed problem grows linearly with the number of sensors, deriving a closed form solution for it is still quite involved. Therefore, we propose an algorithm with a reasonable complexity to find a suboptimal set of transmit powers.

The performance of our proposed suboptimal solution is investigated via a set of numerical examples. The simulation results verify that our proposed power allocation technique results in a performance improvement over full power transmission. This improvement is more pronounced for smaller number of sensors and higher SNRs.

The paper is organized as follows. First, we present the system model and some preliminary materials in Section 2. The achievable data rates of AF MWR are studied in Section 3. The proposed power allocation schemes are discussed in Section 4 and numerical results for its performance are presented in Section 5. At the end, Section 6 concluded the paper.

\section{System Model}

Here, we assume a WSN with $N \geq 2$ sensors denoted by $s_{1}, s_{2}, \ldots, s_{N}$. The sensors want to periodically share their data, with a period called a time slot, for at least $T$ time slots to accomplish a common task. In-network processing at the sensors and network clustering are example situations where such setup may appear. Note that $N$ does not represent the total number of sensors in the network.

To enforce fairness between the sensors, all sensors share data with a common rate $R_{i}^{c}$ during the $i$ th time slot. The common rate is the data rate that all sensors can reliably transmit and receive data with. More formally, if $R_{i, j}$ denotes the achievable transmission rate by $s_{j}$ in the $i$ th time slot, then, $R_{i}^{c}$ is

$$
R_{i}^{c}=\min \left\{R_{i, 1}, R_{i, 2}, \ldots, R_{i, N}\right\}
$$

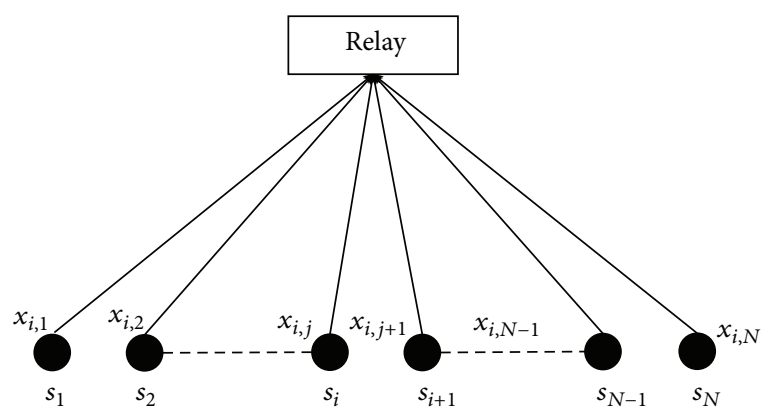

(a) Uplink phase

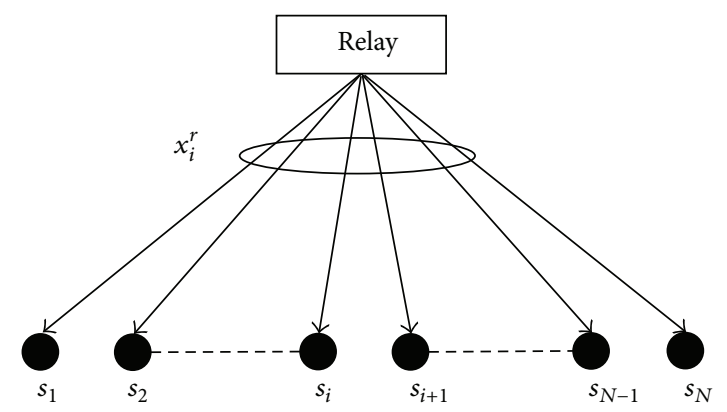

(b) Downlink phase

FIGURE 1: Demonstration of the uplink and downlink phases.

The data sharing sum rate is defined using $R_{i}^{c}$ and denotes the overall rate of data sharing between the sensors over a time span. More specifically, the data sharing sum rate until the $i$ th time slot is defined as

$$
R_{i}^{s}=\sum_{k=1}^{i} \sum_{j=1}^{N} R_{k}^{c}=N \sum_{k=1}^{i} R_{k}^{c} .
$$

2.1. Data Communication Model. In this work, we assume that the sensors do not have a direct link for communication. This could be a result of a harsh environment with surrounding obstacles limiting the direct communication. For such scenario, we suggest using MWR to facilitate data sharing between the sensors. To this end, a relay (central) node is used to help the sensors with their data communication using AF MWR strategy. The relay node could be a sensor serving as a cluster head or a representative for a group of sensors or a special node with more capabilities than the ordinary sensors.

To perform MWR, the time slot is equally divided into two phases, namely, uplink and downlink. In the uplink phase, all sensors simultaneously transmit their messages to the relay node (Figure 1(a)). Hence, the relay receives a superposition of the sensors transmitted signals often referred to as physically network coded signal [12]. Then, the relay amplifies the received signal and broadcasts it to the sensors in the downlink phase (Figure 1(b)). After receiving the relay's broadcast signal, each sensor removes self-interference from the broadcast message and decodes the data of all other sensors. For further information on MWR and how it can help improving the data rate compared to conventional one-way relaying, see $[11,15]$. 
The receiver noise at the relay and the sensors is assumed to be additive white Gaussian with zero mean and unit variance. Further, the channels between the sensors and the relay are reciprocal and the channel gain is fixed for one uplink or downlink phase. For $s_{j}, h_{i, j}$ refers to the channel gain between $s_{j}$ and the relay during the $i$ th time slot.

2.2. Energy Harvesting/Consumption Model. To provide the power needed for data communication, sensors are equipped with a battery with initial energy $E^{i}$ Joules. In addition, during each time slot, sensors harvest energy to further supply power resource for data communication. More specifically, each sensor is capable of harvesting energy with rate $P^{h}$. Please note that the focus of this work is on the energy consumption for data transmission and the energy needed for sending and receiving by the sensors is out of our scope. However, they can be readily incorporated in our analysis.

Here, the transmit power of $s_{j}$ at the $i$ th round, $1 \leq i \leq$ $T$, is denoted by $P_{i, j}$ and the transmit power of the relay is assumed to be $P^{r}$. To guarantee network functionality until $T$, $s_{j}$ takes a conservative approach and limits $P_{i, j}$ such that $P_{i, j} \leq$ $E^{i} / T+P^{h}$. This guarantees that the sensor has enough energy for data sharing at time $T$ even if the harvested energy is negligible.

\section{Deriving $R_{i}^{c}$ for AF MWR}

In this section, we first present the formal signaling model for AF MWR. Then, we study the achievable common rate of data sharing over an arbitrary time slot; say the $i$ th one. Using this analysis, we are able to determine the sum rate of data sharing. Without loss of generality, it is assumed that $\left|h_{i, 1}\right| \leq\left|h_{i, 2}\right| \leq \cdots \leq\left|h_{i, N}\right|$.

After transmission by all sensors in the uplink phase of $i$ th time slot, relay receives the following superimposed signal as depicted in Figure 1(a):

$$
y_{i}^{r}=\sum_{n=1}^{N} h_{i, n} x_{i, n}+n^{r}
$$

where $x_{i, n}$ 's are sensors' messages in the $i$ th time slot and $n^{r}$ is the Gaussian noise at the relay. Now, the relay forms its message, $x_{i}^{r}$, as $x_{i}^{r}=\alpha_{i} y_{i}^{r}$ where

$$
\alpha_{i} \leq \sqrt{\frac{P^{r}}{\sum_{n=1}^{N} P_{i, n}\left|h_{i, n}\right|^{2}+1}}
$$

and broadcasts it to the sensors in the downlink phase as depicted in Figure 1(b). Each sensor then removes its selfinterference from the broadcast signal. After doing such, the received signal at $s_{j}$ is

$$
y_{i, j}=\alpha_{i} h_{i, j} \sum_{n \neq j} h_{i, n} x_{i, n}+\alpha_{i} h_{i, j} n^{r}+n_{j},
$$

where $n_{j}$ is the receiver noise at $s_{j}$. The signal model in (5) is basically similar to the one of a multiple-access (MAC) channel [17]. In this MAC channel, $s_{j}$ wants to decode the message of all other sensors, where $\alpha_{i} h_{i, j} n^{r}+n_{j}$ is the effective noise. Achievable data rates for fading MAC channels have been studied in prior studies, for example, [18]. Please note that in conventional MAC channels, unlike MWR, there is only one communication hop between the sources and the destination. That said, the results in [18] can still be used to find achievable data rates in our WSN setup. More specifically, it can be shown that the data of all other sensors can be reliably decoded at $s_{j}$ if their transmit data rates satisfy the following constraint:

$$
\sum_{n \in \mathcal{S}_{j}} R_{i, n} \leq \frac{1}{2} \log _{2}\left(1+\frac{\alpha_{i}^{2}\left|h_{i, j}\right|^{2} \sum_{n \in \mathcal{S}_{j}} P_{i, n}\left|h_{i, n}\right|^{2}}{1+\alpha_{i}^{2}\left|h_{i, j}\right|^{2}}\right),
$$

for all nonempty $\mathcal{S}_{j} \subseteq\{1,2, \ldots, N\} \backslash j$. Note that for decoding other sensors' messages, each sensor should have the knowledge of all channel gains. The relay node can obtain this information via pilot signaling and then pass it to the sensors. Looking at (6), one can identify another difference between conventional MAC channels and the considered WSN with MWR. Here, the achievable data rates at the receiver, $s_{j}$, depend not only on the other users' transmit power but also on $s_{j}$ transmit power through $\alpha_{i}$. This in turn leads to significant interdependency between the allocation of the users' transmit powers and the users' achievable data rates.

To find the achievable rate at other sensors, one can follow a model similar to (5). As a result, for a rate tuple $\mathscr{R}=$ $\left(R_{i, 1}, R_{i, 2}, \ldots, R_{i, N}\right)$ to be achievable at all sensors during time slot $i, N$ constraints similar to (6) should be satisfied by $\mathscr{R}$. Since (6) in fact refers to $2^{N-1}-1$ constraints at $s_{j}$ to cover all possible nonempty $\mathcal{S}_{j}$, this means that the rate tuple $\mathscr{R}$ is achievable if $N\left(2^{N-1}-1\right)$ constraints are satisfied.

Before finding the common rate for data sharing in the $i$ th time slot, let us define $R_{i, j}^{c}$ as the achievable common rate at $s_{j}$ referring to the rate that all other sensors can reliably transmit their data to $s_{j}$ with. To find $R_{i, j}^{c}$, we set all $R_{i, n}$ in (6) equal to $R_{i, j}^{c}$ resulting in $\sum_{n \in \mathcal{S}_{j}} R_{i, n}=\left|\mathcal{S}_{j}\right| R_{i, j}^{c}$, where $\left|\mathcal{S}_{j}\right|$ is the cardinality of $\mathcal{S}_{j}$. Thus,

$$
R_{i, j}^{c} \leq \min _{\mathcal{S}_{j}} R_{i, j}^{\mathcal{S}_{j}},
$$

where

$$
R_{i, j}^{\mathcal{S}_{j}}=\frac{1}{2\left|\mathcal{S}_{j}\right|} \log _{2}\left(1+\frac{\alpha_{i}^{2}\left|h_{i, j}\right|^{2} \sum_{n \in \mathcal{S}_{j}} P_{i, n}\left|h_{i, n}\right|^{2}}{1+\alpha_{i}^{2}\left|h_{i, j}\right|^{2}}\right) .
$$

Now, using the common rate definition (1), we have

$$
R_{i}^{c} \leq \min _{j} \min _{\mathcal{S}_{j}} \frac{1}{2\left|\mathcal{S}_{j}\right|} \log _{2}\left(1+\frac{\alpha_{i}^{2}\left|h_{i, j}\right|^{2} \sum_{n \in \mathcal{S}_{j}} P_{i, n}\left|h_{i, n}\right|^{2}}{1+\alpha_{i}^{2}\left|h_{i, j}\right|^{2}}\right) .
$$

Note that for a given set of power at the sensors, the common rate is maximum when the condition in (9) holds with equality. Furthermore, we have the following propositions based on our analysis. 
Proposition 1. $R_{i}^{c}$ is an increasing functions of $\alpha_{i}$ and as a consequence an increasing function of $P^{r}$.

In the rest of the paper, we assume that the relay injects enough power into the broadcast signal such that (4) is satisfied with equality to increase the data sharing rate.

Proposition 2. For any $j, R_{i, j}^{c}$ is an increasing function of $P_{i, n}$ for all $n \neq j$ and a decreasing function of $P_{i, j}$.

As we discussed before, finding $R_{i}^{c}$ originally requires checking $N\left(2^{N-1}-1\right)$ constraints. However, these conditions are not independent and all of them depend on $h_{i, 1}, h_{i, 2}, \ldots, h_{i, N}$ and the sensors transmit power. The following theorem indicates how their dependency can be exploited to reduce the number of conditions to be checked for finding $R_{i}^{c}$. This, as a result, decreases the complexity of calculating the sum rate.

Theorem 3. For any arbitrary sensor $s_{j}, R_{i, j}^{c}$ is bounded by those $R_{i, j}^{\mathcal{S}_{j}}$ 's where $\left|\mathcal{S}_{j}\right| \geq j-1$ and $\{1,2, \ldots, j-1\} \subseteq \mathcal{S}_{j}$.

Proof. See Appendix.

Corollary 4. $R_{i}^{c}$ can be found by checking only $2^{N}-2$ constraints rather than all possible $N\left(2^{N-1}-1\right)$ constraints.

Proof. See Appendix.

Having the achievable common rate of each time slot, the achievable data sharing sum rate is derived using (2).

\section{Rate-Improving Power Allocation for AF MWR}

As discussed earlier, the achievable common rate within each time slot and consequently the overall sum rate depend on the sensors' transmit power. Thus, it is desired to seek for a power allocation at the sensors that give the best performance, meaning achieving the highest data sharing sum rate, while keeping the network operational for at least $T$ time slots. The following theorem helps us to understand the features of such power allocation.

Theorem 5. Full power transmission at the sensors, that is, $P_{i, j}=E^{i} / T+P^{h}$ for all $s_{j}$ and $i$, does not guarantee achieving maximum $R_{i}^{c}$.

Proof. See Appendix.

The point stated in Theorem 5 is different from what we see in conventional cooperative data sharing schemes based on one-way relaying and time sharing. That is, while full power transmission guarantees maximum data rate in conventional cooperative schemes, Theorem 5 implies that we can use an MWR strategy where sensors do not transmit with full power and gain a twofold benefit. That is to improve both data rate and energy efficiency compared to an MWR strategy sending with full power at the sensors. This gives a guideline to further improve MWR performance for WSN applications. Now, the question is if full power transmission does not give the best performance, what power allocation reaches the best performance, that is highest sum rate while achieving a lifetime of at least $T$ time slots.

To address the above question, we formulate an optimization problem. The goal of this optimization problem is to maximize the sum rate while guaranteeing that the network lifetime surpasses $T$ time slots as follows:

$$
\begin{gathered}
\max _{P_{1,1}, \ldots, P_{\ell, N}} R_{\ell}^{s}, \\
R_{\ell}^{s}=N \sum_{i=1}^{\ell} R_{i}^{c}, \\
R_{i}^{c} \leq \min _{j} \min _{\mathcal{S}_{j}} \frac{1}{2\left|\mathcal{S}_{j}\right|} \log _{2}\left(1+\frac{\alpha_{i}^{2}\left|h_{i, j}\right|^{2} \sum_{n \in \mathcal{S}_{j}} P_{i, n}\left|h_{i, n}\right|^{2}}{1+\alpha_{i}^{2}\left|h_{i, j}\right|^{2}}\right), \\
\sum_{k=1}^{i} P_{k, j} \leq E^{i}+i P^{h}, \quad \forall i \leq \ell, \forall j \in\{1, \ldots, N\}, \\
0 \leq P_{k, j}, \quad \forall k \leq \ell, \forall j \in\{1, \ldots, N\} .
\end{gathered}
$$

In this optimization problem, the constraint in (13) ensures that the network lifetime is at least $T$ time slots. Also, (14) guarantees that the sensors' consumed energy for data transmission at any time slot is always less than the sum of the energy provided by the initial battery and the harvested energy.

Based on the statement in Theorem 3, the above optimization problem has at least $2^{N}+2 N T$ constraints that grow exponentially with the number of sensors. That said, the optimization problem is quite difficult to solve especially for large $N$. In the following, we discuss how one can find a suboptimal solution to the optimization problem in (10) with significantly lower complexity.

4.1. Relaxing Problem. As we discussed in Section 2, an arbitrary sensor $s_{j}$ limits its transmit power such that $P_{i, j} \leq E^{i} / T+$ $P^{h}$. This guarantees that the sensor never runs out of power before $T$. Using this point, we relax the optimization problem in (10) and rather focus on maximizing $R_{i}^{c}$ for any arbitrary $i \leq$ $T$ assuming $P_{i, j} \leq E^{i} / T+P^{h}$. Solving this relaxed optimization problem for all time slots, $i \leq T$ can give a suboptimal solution for the original sum rate maximization problem. To this end, we formulate an optimization problem to maximize the common rate at time slot $i, R_{i}^{c}$, as follows:

$$
\begin{gathered}
\max _{P_{i, 1}, \ldots, P_{i, N}} \min R_{i, j}^{c}, \\
P_{i, j} \leq \frac{E^{i}}{T}+P^{h}, \quad \forall j, \\
0 \leq P_{i, j}, \quad \forall j .
\end{gathered}
$$


(1) Assign full power to all sensors by setting $P_{i, j}=\left(E^{i} / T\right)+P^{h}$

(2) Choose a power deduction step $\epsilon_{p}$

(3) Set $B=\{\}$

(4) repeat

(5) Calculate $R_{i, j}^{c}$ for all $j$ based on the relaxed constraints

(6) Find $R_{i}^{c}=\min R_{i, j}^{c}$ and $k=\operatorname{argmin} R_{i, j}^{c}$

(7) $B=B \cup\{k\}$

(8) For all $j \in B, P_{i, j}=P_{i, j}-\epsilon_{p}$

(9) until $R_{i}^{c}$ shows no improvement compared to the previous step

Algorithm 1: Algorithmic presentation of the proposed power allocation technique. into

Using Fact 1, the above max-min problem is transformed

$$
\begin{gathered}
\max _{P_{i, 1}, \ldots, P_{i, N}} R_{i}^{\text {th }}, \\
\frac{P^{r}\left|h_{i, j}\right|^{2} \sum_{n \in \mathcal{S}_{j}} P_{i, n}\left|h_{i, n}\right|^{2}}{P^{r}\left|h_{i, j}\right|^{2}+\sum_{n=1}^{N} P_{i, n}\left|h_{i, n}\right|^{2}+1} \geq 2^{2\left|\mathcal{S}_{j}\right| R_{i}^{\text {th }}}-1 \quad \forall j, \mathcal{S}_{j}, \\
P_{i, j} \leq \frac{E^{i}}{T}+P^{h}, \quad \forall j, \\
0 \leq P_{i, j}, \quad \forall j .
\end{gathered}
$$

The number of constraints in (17) still grows exponentially with the number of sensors. To this end, we further simplify rate maximization problem by relaxing some of the rate constraints. More specifically, we consider the rate constraints, where $\left|\delta_{j}\right|=N-1$ for any $j$, that is, $N-1$-tuple constraints. These constraints have the smallest prelog factor, that is, $1 /(N-1)$, among constraints presented in (6). Thus, in medium to high SNR range, they are more likely to limit $R_{i}^{c}$. Without loss of generality, one could also assume that during the $i$ th time slot, $\left|h_{i, 1}\right| \leq\left|h_{i, 2}\right| \leq \cdots \leq\left|h_{i, N}\right|$. The relaxed problem also considers all single rate constraints at the worst user, that is, $s_{1}$, and the single rate constraint on the data rate of the worst user at the second worst user, that is, $R_{i, 1}$ at $s_{2}$, as follows:

$$
\begin{gathered}
\max _{P_{i, 1}, \ldots, P_{i, N}} R_{i}^{\text {th }} \\
\frac{P^{r} h_{i, j}^{2} \sum_{n=1, n \neq j}^{N} P_{i, n}\left|h_{i, n}\right|^{2}}{P^{r}\left|h_{i, j}\right|^{2}+\sum_{n=1}^{N} P_{i, n}\left|h_{i, n}\right|^{2}+1} \geq 2^{2(N-1) R_{i}^{\text {th }}}-1 \quad \forall j, \\
\frac{P^{r}\left|h_{i, 1}\right|^{2} P_{i, j}\left|h_{i, j}\right|^{2}}{P^{r}\left|h_{i, 1}\right|^{2}+\sum_{n=1}^{N} P_{i, n}\left|h_{i, n}\right|^{2}+1} \geq 2^{2(N-1) R_{i}^{\text {th }}}-1 \quad \forall j \neq 1, \\
\frac{P^{r}\left|h_{i, 2}\right|^{2} P_{i, 1}\left|h_{i, 1}\right|^{2}}{P^{r}\left|h_{i, 2}\right|^{2}+\sum_{n=1}^{N} P_{i, n}\left|h_{i, n}\right|^{2}+1} \geq 2^{2(N-1) R_{i}^{\text {th }}-1} \\
P_{i, j} \leq \frac{E^{i}}{T}+P^{h}, \quad \forall j \\
0 \leq P_{i, j}, \quad \forall j .
\end{gathered}
$$

To understand the intuition behind considering (21), recall that, for any $k \neq 1$, when $\mathcal{S}_{k}=\{j\}$ and $j \notin\{1, k\}$, one can show that $R_{i, k}^{\mathcal{S}_{k}} \geq R_{i, 1}^{\mathcal{S}_{1}}$, where $\mathcal{S}_{1}=\{j\}$. As a result, if (21) is satisfied, it is ensured that the smallest bound on $s_{j}^{\prime}$ s single rate for any $2 \leq j$ has been taken into account. In a similar way, one can argue that (22) represents the smallest bound on the $s_{1}^{\prime}$ s single rate. Thus, the relaxed optimization problem in (19) considers all $N-1$-tuple rate constraints as well as the worst (smallest) bounds on the single rates. This relaxation significantly decreases the number of constraints to $4 \mathrm{~N}$ which grows linearly with the number of sensors.

4.2. Proposed Suboptimal Solution for the Relaxed Problem. Although the relaxed optimization problem has significantly less number of constraints compared to the original problem, finding an analytical solution for it is quite involved. To address this challenge, we suggest a method to find a numerical suboptimal solution for the relaxed problem. The logic behind the proposed numerical solution is similar to the approach taken in the proof of Theorem 5.

The proposed algorithm for finding suboptimal power allocation is presented in Algorithm 1. The proposed algorithm starts from an initial point for power allocations by assuming full power transmission for all sensors and then uses the point stated in Proposition 2 to further improve the rate. To this end, we find $k=\operatorname{argmin}_{j} R_{i, j}^{c}$, where $R_{i, j}^{c}$ 's are calculated based on merely the relaxed constraints, that is, single rate constraints at $s_{1}$ and the constraint on $s_{1}$ 's rate at $s_{2}$ plus all $N-1$-tuple constraints. We name $s_{k}$ the bottleneck sensor since it limits the common rate $R_{i}^{c}$. Now, based on the statement in Proposition $2, R_{i, k}^{c}$ is a decreasing function of $P_{i, k}$. Thus, if we back off $P_{i, k}$, say with step $\epsilon_{p}, R_{i, k}^{c}$ sees an increase at the cost of a rate decrease for all other sensors. Backing off $P_{i, k}$ continues to the point where $s_{k}$ is not bottleneck anymore and another sensor, for example, $s_{l}$, limits the rate and becomes the bottleneck. Now, if $s_{l}$ backs off its power to increase $R_{i, l}^{c}, R_{i, k}^{c}$ sees a decline. However, if the transmit power at both $s_{k}$ and $s_{l}$ decreases with step $\epsilon_{p}, R_{i, k}^{c}$ and $R_{i, l}^{c}$ could both see an increase. Backing off the power at $s_{k}$ and $s_{l}$ continues till another sensor becomes the bottleneck. The algorithm continues looking for bottleneck sensors and backing off their power up to the point where no further improvement at $R_{i}^{c}$ can be achieved. Note that, in Algorithm 1, $B$ is a set containing all sensors that have been a bottleneck 
sensor at some point. In our proposed algorithm, the power allocation at the sensors always moves in a direction where $R_{i}^{c}$, calculated based on the relaxed constraints, increases. As a result, the algorithms climb up toward local maxima.

When the algorithm stops, it gives a set of power allocation denoted by $\left(P_{i, 1}^{*}, P_{i, 2}^{*}, \ldots, P_{i, N}^{*}\right)$ giving a suboptimal value for $R_{i}^{c}$ when only relaxed constraints are considered. This is not, however, the actual value for the common rate in time slot $i$. The actual $R_{i}^{c}$ is found by considering all $2^{N}-2$ rate constraints with $\left(P_{i, 1}^{*}, P_{i, 2}^{*}, \ldots, P_{i, N}^{*}\right)$ as the set of transmit powers at the sensors. Please note that there is a performance gap between the data rate achieved through the power allocation given by the suboptimal algorithm and the optimal solution. However, finding the optimal solution has an exponential complexity compared to the linear complexity of the proposed suboptimal algorithm. Thus, finding the optimal solution is not feasible in a reasonable time.

At time slot $i$ and for an arbitrary $s_{j}, a_{i, j}=P_{i, j}^{*}-E^{i} / T-P^{h}$ represents the energy saving that is achieved through our proposed power allocation. If $a_{i, j}>0, s_{j}$ stores the saved energy. At the end of $T$ time slots, the total saved energy is $A_{j}=\sum_{i=1}^{T} a_{i, j}$. Using this energy, $s_{j}$ extends its lifetime beyond $T$ and continues sharing data with other sensors in the same fashion as up to $T$. Let us denote the time slot when the first sensor runs out of energy by $T^{*} \geq T$. Now, the overall sum rate of AF MWR with our proposed power allocation is

$$
R_{T^{*}}^{s}=N \sum_{i=1}^{T^{*}} R_{i}^{c} .
$$

Note that when full power transmission is in effect, all sensors deplete their batteries and run out of energy at the end of the $T$ th time slot.

\section{Simulation Results}

In this section, we present several numerical examples to evaluate the performance of our proposed power allocation. To this end, we compare the performance of AF MWR with the proposed power allocation with the performance of AF MWR with full power transmission. In our simulation results, it is assumed that the channel gains between the sensors and the relay follow a Rayleigh distribution with average power 1 . To take into account the effect of channel gains randomness, the results are averaged over 100 realizations for $T=50$. For a given transmit SNR, we set $E^{i}=10^{\mathrm{SNR} / 10} T$ to ensure transmitting with the specified SNR over all time slots. In addition, $P^{r}=10^{\mathrm{SNR} / 10}$ and $\epsilon_{p}=0.001 \mathrm{SNR}$. Note that, here, we present actual rates by checking all $2^{N}-2$ constraints for the set of transmit powers.

Figure 2 depicts the normalized sum rate of AF MWR with our proposed allocation for different values of $N$. In this figure, $P^{h}=10 \mathrm{~dB}$ and the normalized rate is derived by dividing the sum rate of our proposed technique to that of full power transmission. As seen, the improvement is more pronounced for larger SNRs and smaller $N$. It reaches around 18 percent for $N=3$ and $\mathrm{SNR}=30 \mathrm{~dB}$. The reason is that, for

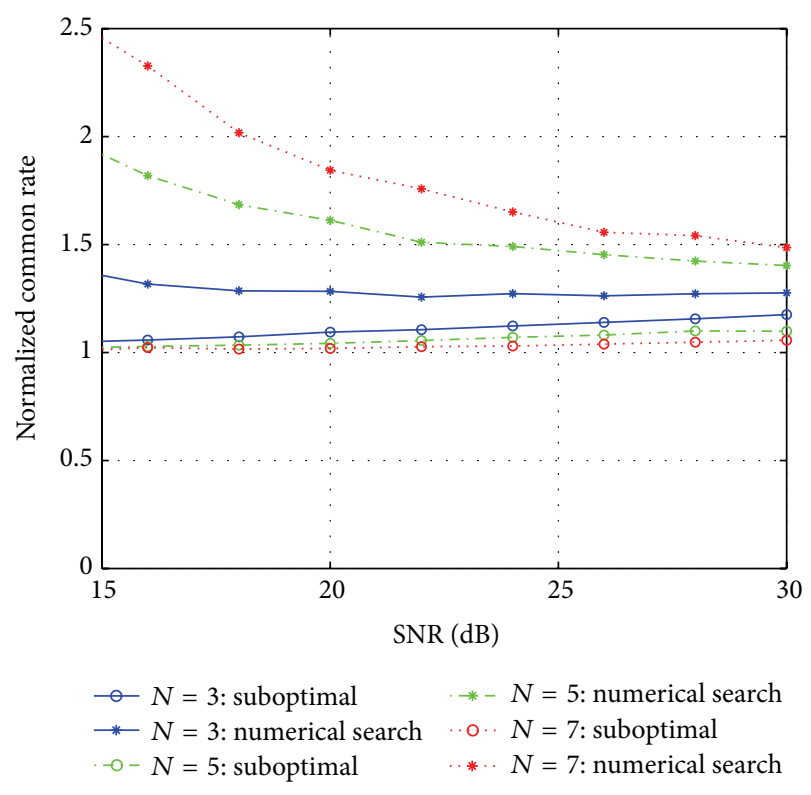

FIgURE 2: Normalized achievable common rate over SNR.

smaller SNRs, it is more likely that the constraints omitted in the relaxed problem actually limit the data rate.

In addition, in this figure, we compare our suboptimal results with the numerical solution for the original (nonrelaxed) optimization problem presented in (10)-(15). To find the numerical solution, we have used a Monte Carlo search method during each time slot. More specifically, for time slot $i$, we generate 1000 random $N$-tuples for $\left(P_{i, 1}, P_{i, 2}, \ldots, P_{i, N}\right)$. Then, after checking all rate conditions, the $N$-tuple that results in the maximum $R_{i}^{c}$ is chosen as the optimal solution. As seen in Figure 2, there is a significant gap between the Monte Carlo numerical solution and our proposed suboptimal solution. However, one should keep in mind that this improvement comes at the price of significantly longer simulation run time. The performance gap between the suboptimal solution and the numerical solution decreases with increasing SNR since the constraints considered in the relaxed optimization problem are more likely to be active in the original optimization problem as well.

The effect of the number of sensors on the performance of the proposed power allocation is captured with more detail in Figure 3. As seen, the proposed power allocation results in a more noticeable improvement for smaller $N$. Note that when $N$ increases, it is more likely to have a small channel gain for one of the links limiting the data rate through constraints not considered in the relaxed optimization problem.

Figure 4 presents the normalized rate of the proposed power allocation versus the energy harvest rate $P^{h}$. Higher $P^{h}$ means having more power resources available for transmission that in turn increases the effective SNR, that is, provided by the initial battery and the harvested power. As a result, we observe a similar behavior to Figure 2 where rate improvement is more visible for higher SNRs. 


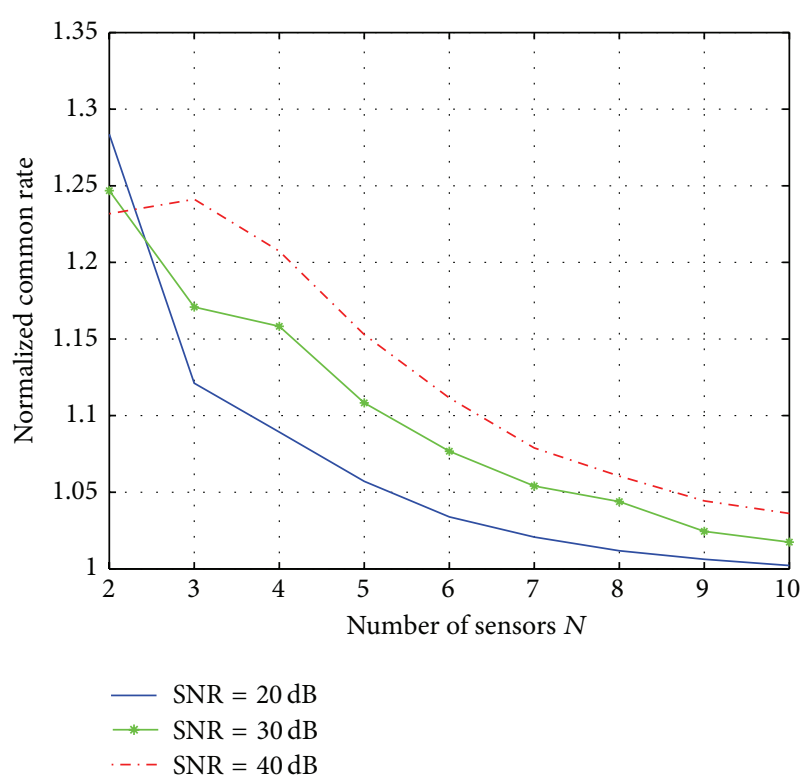

FIGURE 3: Normalized achievable common rate of the suboptimal solution for different $N$.

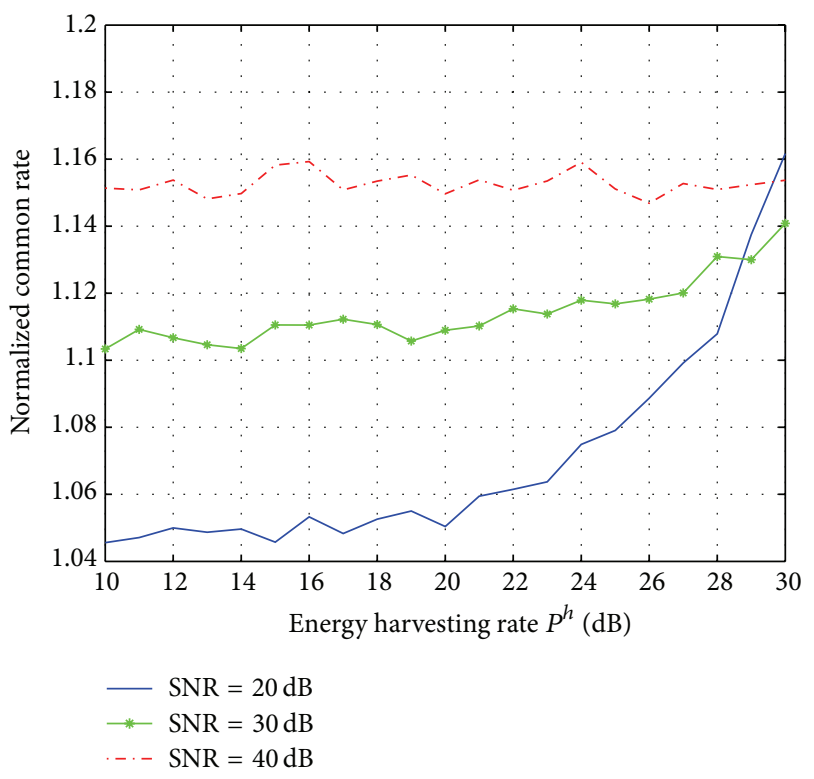

FIgURE 4: Normalized achievable common rate of the suboptimal solution for different energy harvesting rate $P^{h}$.

\section{Conclusion}

In this paper, we suggested the application of MWR for data sharing in an energy harvesting WSN. First, we studied the achievable common data rate for each round of data sharing and the overall data sharing sum rate between the sensors. Using our rate study, we then formulated an optimization problem to maximize the data sharing sum rate while a desired network lifetime is assured. Due to the complexity of this optimization problem, we then suggested a relaxed version of the problem to adjust the power allocation at the sensors for an improved data sharing sum rate. Then, a suboptimal solution for the relaxed optimization problem was proposed. Simulations results verified that our suboptimal solution indeed enhances the data sharing sum rate. The level of the rate improvement depends on the number of sensors as well as SNR. Alternatively, one can use MWR and focus on extending the lifetime rather than the data rate. To solve this problem, an approach similar to the proposed power allocation in this paper can be taken.

\section{Appendix}

Proof of Theorem 3. For $s_{1}$, the theorem statement is true since it means checking all constraints at $s_{1}$. Now, consider the constraints at $s_{j}$ for $j \geq 2$. If $\left|\mathcal{S}_{j}\right|<j-1$, then $\exists k<j$ where $k \notin \mathcal{S}_{j}$ and there exists an $\mathcal{S}_{k}$ such that $\mathcal{S}_{k}=\mathcal{S}_{j}$. Now, remember that $\left|h_{k}\right| \leq\left|h_{j}\right|$; hence, $R_{i, k}^{\mathcal{S}_{k}} \leq R_{i, j}^{\mathcal{S}_{j}}$. This ensures that $R_{i, j}^{\mathcal{S}_{j}}$ does not limit the common rate. As a result, checking its associated rate condition is unnecessary. Now, assume that $\left|\mathcal{S}_{j}\right| \geq j-1$ and $\exists k \in\{1,2, \ldots, j-1\}$, where $k \notin \mathcal{S}_{j}$. With a similar argument, one can show that there is an $\mathcal{S}_{k}$ such that $R_{i, k}^{\mathcal{S}_{k}} \leq R_{i, j}^{\mathcal{S}_{j}}$. Therefore, checking condition associated with $\mathcal{S}_{j}$ is unnecessary. This completes the proof.

Proof of Corollary 4. Using Theorem 3, at any arbitrary sensor $s_{j}$, one can find $R_{i, j}^{c}$ by checking only

$$
c_{j}=\sum_{k=0}^{k=N-j}\left(\begin{array}{c}
N-j \\
k
\end{array}\right)=2^{N-j}
$$

constraints. Thus, the total number of constraints to be checked at all sensors is

$$
C=\sum_{j=1}^{N} c_{j}=\sum_{j=1}^{N} 2^{N-j}=2^{N}-2 .
$$

Proof of Theorem 5. Assume we assign full transmit power to all sensors and the channel gains are such that for $k \neq l$, $R_{i, k}^{c} \neq R_{i, l}^{c}$. For full power transmission, the system achieves the common rate of $R_{F}^{c}$. From the definition of common rate in (9), $R_{F}^{c}=R_{i, j}^{c}$ where $j=\operatorname{argmin} R_{i, n}^{c}$. Now, using Fact 2, $s_{j}$ can reduce its power to increase $R_{i, j}^{c}$ and decrease the minimum achievable common rate of all other sensors, that is, $\min _{n \neq j} R_{i, n}^{c}$, to the point where $R_{i, j}^{c}=\min _{n \neq j} R_{i, n}^{c}$. This means that the common rate of the assumed MWRC increases and surpasses $R_{F}^{c}$. Thus, $R_{F}^{c}$ is not the maximum achievable common rate.

\section{Conflict of Interests}

The authors declare that there is no conflict of interests regarding the publication of this paper. 


\section{Acknowledgments}

The authors would like to thank Alberta Innovates Technology Futures (AITF) and Natural Sciences and Engineering Research Council of Canada (NSERC) for supporting their research.

\section{References}

[1] J. Kulik, W. Heinzelman, and H. Balakrishnan, "Negotiationbased protocols for disseminating information in wireless sensor networks," Wireless Networks, vol. 8, no. 2-3, pp. 169-185, 2002.

[2] C.-Y. Chong and S. P. Kumar, "Sensor networks: evolution, opportunities, and challenges," Proceedings of the IEEE, vol. 91, no. 8, pp. 1247-1256, 2003.

[3] D. Blatt and A. Hero, "Distributed maximum likelihood estimation for sensor networks," in Proceedings of the IEEE International Conference on Acoustics, Speech, and Signal Processing (ICASSP '04), vol. 3, pp. III929-III932, IEEE, May 2004.

[4] Y.-C. Tseng, S.-Y. Ni, Y.-S. Chen, and J.-P. Sheu, "The broadcast storm problem in a mobile ad hoc network," Wireless Networks, vol. 8, no. 2-3, pp. 153-167, 2002.

[5] S. Cui, A. J. Goldsmith, and A. Bahai, "Energy-efficiency of MIMO and cooperative MIMO techniques in sensor networks," IEEE Journal on Selected Areas in Communications, vol. 22, no. 6, pp. 1089-1098, 2004.

[6] Z. Zhou, S. Zhou, J.-H. Cui, and S. Cui, "Energy-efficient cooperative communication based on power control and selective single-relay in wireless sensor networks," IEEE Transactions on Wireless Communications, vol. 7, no. 8, pp. 3066-3078, 2008.

[7] J. N. Al-Karaki and A. E. Kamal, "Routing techniques in wireless sensor networks: a survey," IEEE Wireless Communications, vol. 11, no. 6, pp. 6-27, 2004.

[8] V. Naik, A. Arora, P. Sinha, and H. Zhang, "Sprinkler: a reliable and energy efficient data dissemination service for extreme scale wireless networks of embedded devices," IEEE Transactions on Mobile Computing, vol. 6, no. 7, pp. 777-789, 2007.

[9] H. Sabbineni and K. Chakrabarty, "Location-aided flooding: an energy-efficient data dissemination protocol for wireless sensor networks," IEEE Transactions on Computers, vol. 54, no. 1, pp. 36-46, 2005.

[10] Z. Zhou, S. Zhou, S. Cui, and J.-H. Cui, "Energy-efficient cooperative communication in a clustered wireless sensor network," IEEE Transactions on Vehicular Technology, vol. 57, no. 6, pp. 3618-3628, 2008.

[11] D. Gundiiz, A. Yener, A. Goldsmith, and H. V. Poor, “The multiway relay channel," in Proceedings of the IEEE International Symposium on Information Theory (ISIT '09), pp. 339-343, July 2009.

[12] B. Nazer and M. Gastpar, "Reliable physical layer network coding," Proceedings of the IEEE, vol. 99, no. 3, pp. 438-460, 2011.

[13] L. Ong, C. M. Kellett, and S. J. Johnson, "Capacity theorems for the AWGN multi-way relay channel," in Proceedings of the IEEE International Symposium on Information Theory (ISIT '10), pp. 664-668, June 2010.

[14] L. Ong, S. J. Johnson, and C. M. Kellett, “The capacity region of multiway relay channels over finite fields with full data exchange," IEEE Transactions on Information Theory, vol. 57, no. 5, pp. 3016-3031, 2011.
[15] M. Noori and M. Ardakani, "On the achievable rates of symmetric Gaussian multi-way relay channels," EURASIP Journal on Wireless Communications and Networking, vol. 2013, no. 1, article 11, 2013.

[16] M. Noori and M. Ardakani, "On the achievable rates of memoryless two-way relay channels," in Proceedings of the IEEE 78th Vehicular Technology Conference (VTC '13), pp. 1-5, September 2013.

[17] T. M. Cover and C. S. Leung, "An achievable rate region for the multiple-access channel with feedback," IEEE Transactions on Information Theory, vol. 27, no. 3, pp. 292-298, 1981.

[18] D. N. Tse and S. V. Hanly, "Multiaccess fading channels. I. Polymatroid structure, optimal resource allocation and throughput capacities," IEEE Transactions on Information Theory, vol. 44, no. 7, pp. 2796-2815, 1998. 

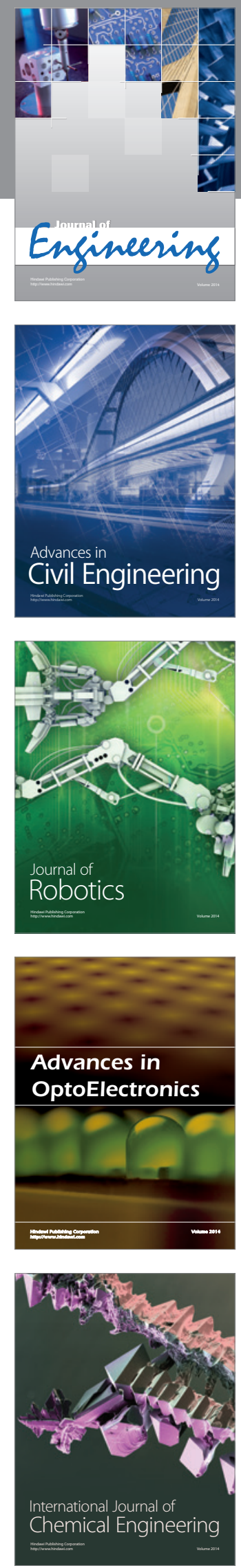

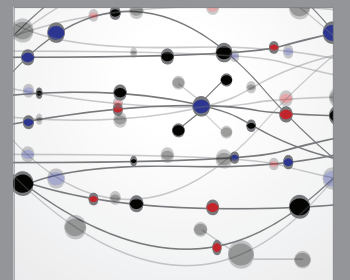

The Scientific World Journal
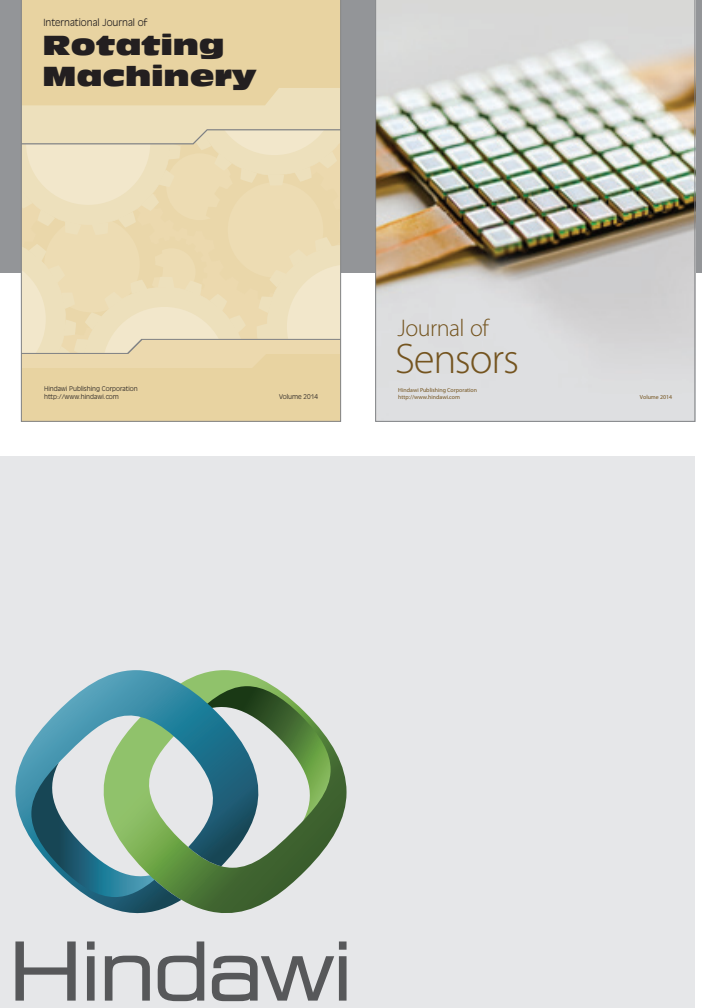

Submit your manuscripts at http://www.hindawi.com
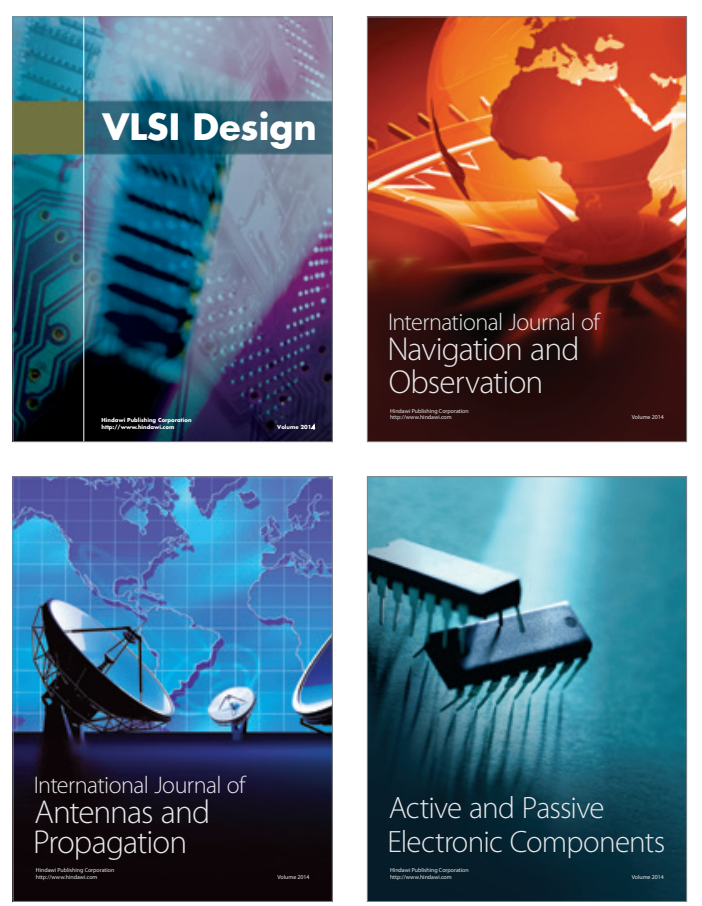
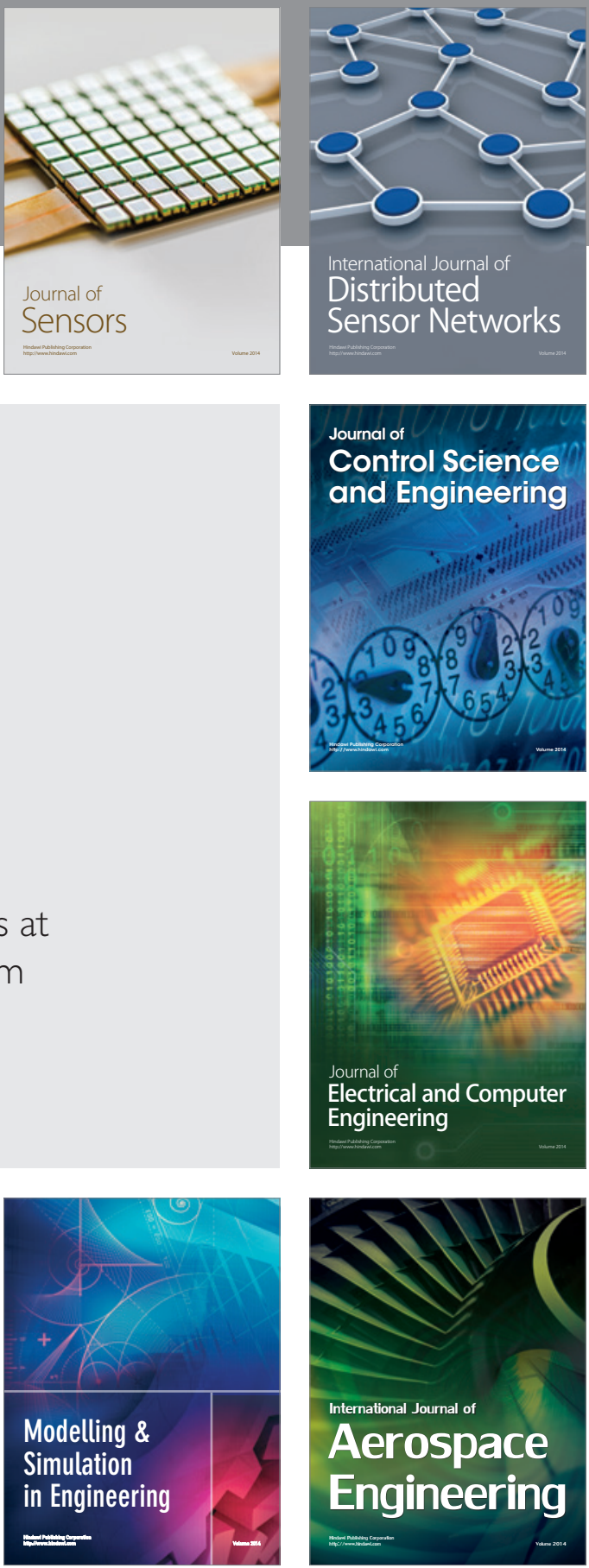

Journal of

Control Science

and Engineering
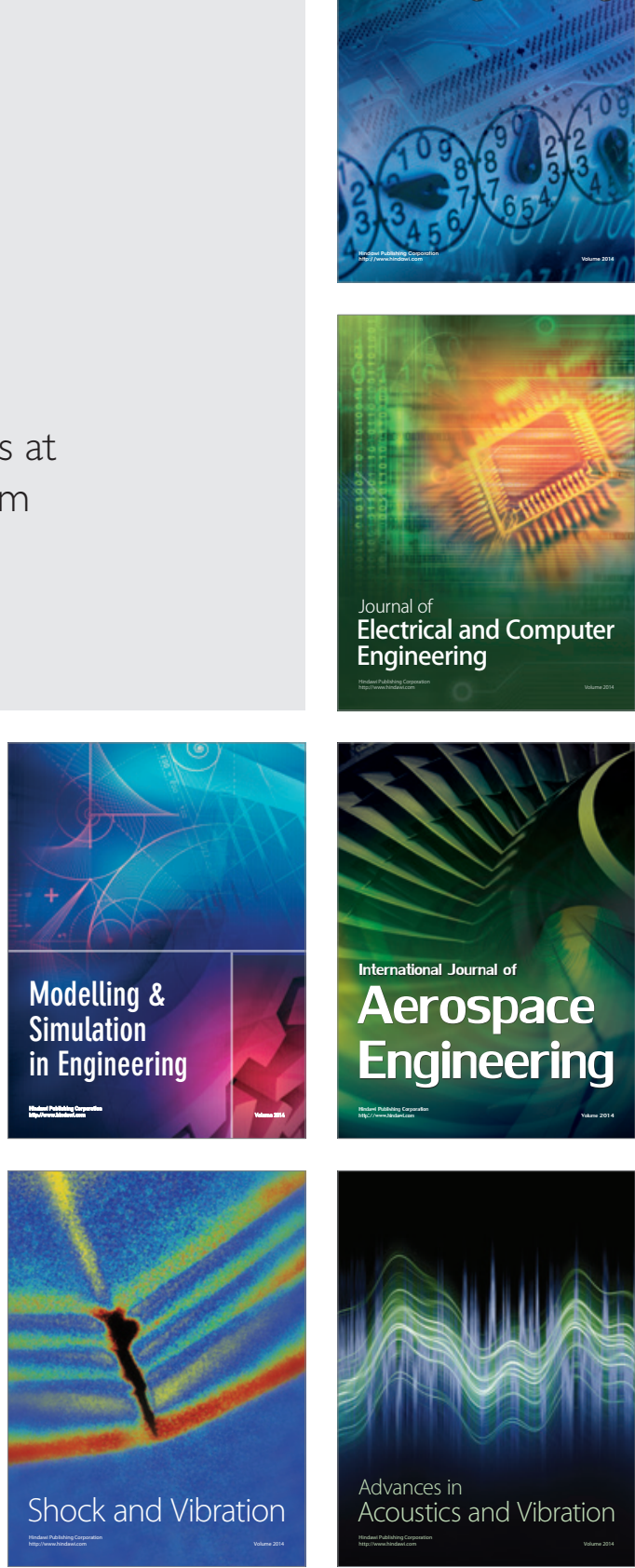